Article

\title{
Expression Profiles of Long Non-Coding RNA GAS5 and MicroRNA-222 in Younger AML Patients
}

\author{
Djordje Pavlovic $^{1}$, Natasa Tosic ${ }^{1}$, Branka Zukic ${ }^{1}$ (D), Zlatko Pravdic ${ }^{2}$, Nada Suvajdzic Vukovic ${ }^{2,3}$, Sonja Pavlovic ${ }^{1}$ \\ and Vladimir Gasic ${ }^{1, *}$ \\ 1 Laboratory for Molecular Biomedicine, Institute of Molecular Genetics and Genetic Engineering, University of \\ Belgrade, 11042 Belgrade, Serbia; djordje5996@gmail.com (D.P.); natasa.tosic@imgge.bg.ac.rs (N.T.); \\ branka.zukic@imgge.bg.ac.rs (B.Z.); sonja.pavlovic99@gmail.com (S.P.) \\ 2 Clinic of Hematology, Clinical Center of Serbia, 11000 Belgrade, Serbia; zlatko.pravdic@gmail.com (Z.P.); \\ suvajdzic.nada@gmail.com (N.S.V.) \\ 3 School of Medicine, University of Belgrade, 11000 Belgrade, Serbia \\ * Correspondence: vlada.gasic42@gmail.com; Tel.: +381-65-3976-445; Fax: +381-11-3975-808
}

check for

updates

Citation: Pavlovic, D.; Tosic, N.;

Zukic, B.; Pravdic, Z.; Vukovic, N.S.; Pavlovic, S.; Gasic, V. Expression Profiles of Long Non-Coding RNA GAS5 and MicroRNA-222 in Younger AML Patients. Diagnostics 2022, 12, 86. https://doi.org/10.3390/ diagnostics 12010086

Academic Editor:

Chung-Che (Jeff) Chang

Received: 14 November 2021

Accepted: 24 December 2021

Published: 30 December 2021

Publisher's Note: MDPI stays neutral with regard to jurisdictional claims in published maps and institutional affiliations.

Copyright: (C) 2021 by the authors. Licensee MDPI, Basel, Switzerland. This article is an open access article distributed under the terms and conditions of the Creative Commons Attribution (CC BY) license (https:// creativecommons.org/licenses/by/ $4.0 /)$.

\begin{abstract}
Acute myeloid leukemia (AML) is a heterogeneous malignant disease both on clinical and genetic levels. AML has poor prognosis and, therefore, there is a constant need to find new prognostic markers, as well as markers that can be used as targets for innovative therapeutics. Recently, the search for new biomarkers has turned researchers' attention towards non-coding RNAs, especially long non-coding RNAs (lncRNAs) and micro RNAs (miRNAs). We investigated the expression level of growth arrest-specific transcript 5 (GAS5) lncRNA in 94 younger AML patients, and also the expression level of miR-222 in a cohort of 39 AML patients with normal karyotype (AML-NK), in order to examine their prognostic potential. Our results showed that GAS5 expression level in AML patients was lower compared to healthy controls. Lower GAS5 expression on diagnosis was related to an adverse prognosis. In the AML-NK group patients had higher expression of miR-222 compared to healthy controls. A synergistic effect of $G A S 5^{\text {low }} / \mathrm{miR}-222^{\text {high }}$ status on disease prognosis was not established. This is the first study focused on examining the GAS5 and miR-222 expression pattern in AML patients. Its initial findings indicate the need for further investigation of these two non-coding RNAs, their potential roles in leukemogenesis, and the prognosis of AML patients.
\end{abstract}

Keywords: AML; GAS5; miR-222

\section{Introduction}

Acute myeloid leukemia (AML) is a hematological malignancy that is characterized by the uncontrolled proliferation and impaired differentiation of early myeloid cells leading to accumulation of immature blast cells in the bone marrow and peripheral blood, thus resulting in hematopoietic failure. AML is the most common acute leukemia in adults, accounting for about $80 \%$ of all cases. It is a neoplastic disorder with very poor prognosis, despite the progress made towards discovering the exact etiology of AML. This is due to the fact that the initial treatment protocol is still based on the classification of the patients into risk groups based on the pretreatment karyotype analysis. However, nearly half of the patients do not have any detectable cytogenetic changes. They are referred to as AML with normal karyotype (AML-NK) patients, categorized into the intermediate risk group. Over the years, new molecular markers have been discovered and some of them, like mutations in fms-related tyrosine kinase-3 (FLT3), nucleophosmin 1 (NPM1), CCAAT/enhancer binding protein alpha (CEBPA) gene and runt-related transcription factor 1 (RUNX1) gene, have been included into the revised World Health Organization (WHO) classification of myeloid neoplasms and acute leukemia, and European LeukemiaNet (ELN) risk classification system $[1,2]$. Nonetheless, there is a constant need for discovery of new 
molecular markers that would lead towards more precise risk stratification and efficient personalized therapeutic protocols.

New high-throughput technologies enabled us to perform transcriptome-wide profiling and to seek new biomarkers of different neoplasms beyond protein-coding genes. Thus, one type of non-coding RNA, long non-coding RNAs (lncRNAs), have been shown to regulate main cell processes like differentiation, proliferation and cell cycle [3]. LncRNAs are defined as transcribed RNA molecules with length over 200 nucleotides. They are not translated into proteins, but are involved in gene regulation, and therefore can be involved in cancer pathology [4].

One of the first lncRNAs that was shown to play a major role in the pathogenesis of cancer is growth arrest-specific transcript 5 (GAS5) [5]. The GAS5 gene, located at the 1q25 locus, is approximately 650 bases in length and is organized in 12 exons and 11 introns. The introns encode 10 box C/D snoRNAs (small nucleolar RNAs) that are involved in methylation processes, and therefore in epigenetic regulation. Exons encode a few splice variants of GAS5 mRNA, but due to the presence of a stop codon, none of them produce proteins, and the transcripts are degraded through nonsense mediated decay (NMD) pathway [6]. Although the expression level of GAS5 is increased during growth arrest by serum deprivation, in many types of cancer it was shown that GAS5 is down-regulated indicating a tumor suppressor function of this lncRNA $[7,8]$.

GAS5 performs its tumor suppressor function by various mechanisms of action. GAS5 has the function of a signal molecule and directly participates in the regulation of the p53 signaling pathway. In that way, decreased expression of GAS5 is associated with cell cycle arrest via increased p53 expression [9]. Also, it can function as a sort of decoy, acting as a molecular "sponge" that binds directly to target RNAs or proteins, thus blocking their downstream functioning [10]. In addition to studying the role of GAS5 in the pathogenesis of cancer, its potential role as a prognostic marker was also investigated. In numerous types of cancer, it has been shown that reduced GAS5 expression was associated with unfavorable clinical and pathological characteristics related to advanced stages of the disease, making it a marker for poor prognosis [11-13].

Although the role of GAS5 deregulation has been studied in different types of solid cancers, its impact on the development and prognosis in leukemias, especially in AML, has rarely been investigated. There are only a few studies that are either based on available AML data in The Cancer Genome Atlas database information, or that are dedicated to the existing polymorphisms in the GAS5 gene and their impact on AML prognosis [14,15]. One study reported that certain variants in the GAS5 gene can represent risk factors for AML genesis [16]. GAS5 is of great importance in other types of leukemia, like in certain types of B-ALL characterized by hyperdiploidy or the presence of TCF3/PBX1 rearrangement, but its significance is related to the treatment of ALL, that is, to the administration of glucocorticoids in therapy protocols [17]. In the case of childhood ALL, it has been shown that the GAS5 expression level can be a predictor of therapy response in the first phase of treatment, remission induction [18].

In addition, one of the main proposed mechanisms with which GAS5 exerts its tumor suppressor role is through acting as an endogenous sponge for the oncogenic miR222 [19]. Overexpression of miR-222 has been widely detected across many malignancies, including AML, and has been associated with tumor stage, tumor type and metastasis [20]. The discovery that GAS5 negatively regulates miR-222 activity was first reported in glioma, and it was later confirmed in gastric cancer and recently in B ALL as well [21-23]. Furthermore, it was shown that decreasing miR-222 expression through GAS5 leads to higher expression of an important miR-222 target, the tumor suppressor PTEN, that plays a significant role in cell growth, apoptosis, and migration [19,22].

This is the first study to analyze GAS5 expression patterns in a cohort of younger AML patients ( $<65$ years) and examine its potential influence on the prognosis of the disease. In addition, we separately investigated prognostic significance of GAS5 expression in AML- 
NK group of patients, taking into account the influence of the miR-222 expression and other already established molecular markers of AML.

\section{Materials and Methods}

\subsection{Patients and Therapy Protocol}

A total of 94 bone marrow samples were collected from younger AML patients ( $<65$ years) diagnosed at the Clinic of Hematology, Clinical Center of Serbia, as well as from 14 healthy controls (bone-marrow donors). A total cohort of AML patients consisted of 75 de novo AML cases, 14 were secondary AML (sAML) patients and 5 were therapyrelated AML (tAML) patients. Research was conducted in accordance with the ethical standards of the World Medical Association's Declaration of Helsinki. The study was approved by the Ethics Committee of the Clinical Center of Serbia, and written informed consent was obtained for all patients. Diagnostics was done based on the cytomorphology, immunophenotype using flow-cytometry and cytogenetic analysis. Cytomorphological analysis implies a marrow or blood blast count of $20 \%$ for the diagnosis of AML, except for AML with recurrent cytogenetic abnormalities. Cytogenetic analysis was undertaken by conventional band methodology, while molecular analysis was done using PCR and RT-PCR, and WHO classification was assigned [1]. Mutational analyses of FLT3 and NPM1 genes were analyzed as previously described [24]. Prognostic classification of AML patients was done according to European LeukemiaNet (ELN) recommendations [2]. All patients received induction chemotherapy with daunorubicin and cytarabine according to the protocol $3+7$, followed by three consolidation cycles of high/intermediate doses of cytarabine [2].

\subsection{Growth Arrest-Specific Transcript 5 (GAS5) and MiR-222 Expression Level Analysis}

Mononuclear cells were isolated from bone marrow samples of AML patients and healthy controls using Ficoll-Paque Plus solution (GE Healthcare, Buckinghamshire, UK) according to the manufacturers' protocol. Mononuclear cells were stored in TRI Reagent (Ambion, Thermo Fisher Scientific, Waltham, MA, USA) and kept at $-80^{\circ} \mathrm{C}$ until RNA isolation. Total RNA was isolated using manufacturer protocol. Concentration and purity of total RNA was determined on NanoVue ${ }^{\mathrm{TM}}$ Plus Spectrophotometer (GE Healthcare, Buckinghamshire, UK).

For the expression analysis of GAS5, one microgram of total RNA was used for the cDNA synthesis using RevertAid Reverse Transcriptase (Thermo Fisher Scientific, Waltham, MA, USA). Real-time PCR was performed on 7900HT Fast Real-Time PCR System (Applied Biosystems, Foster City, CA, USA). We have performed PCR using $1 \mu \mathrm{L}$ of cDNA (20 ng RNA equivalent) with TaqMan ${ }^{\circledR}$ Universal Master Mix II (Applied Biosystems, Foster City, CA, USA), and TaqMan ${ }^{\circledR}$ Gene Expression Assay for GAS5 (Hs03464472_m1 ) and for GAPDH (Hs99999905_m1) as endogenous control (Thermo Fisher Scientific, Waltham, MA, USA). All samples were run in duplicate. Relative quantification analysis was performed using comparative ddCt method, using healthy controls as calibrator, meaning $d d C t=d C t$ sample $-d C$ thealthy control (median). ROC curve analyses using on-line program "Cut-off finder" [25] was applied for the identification of optimal cut-off value for discriminating between $G A S 5^{\text {high }}$ and GAS5 ${ }^{\text {low }}$ expression.

For the expression analysis of miR-222, $60 \mathrm{ng}$ of total RNA was used for the cDNA synthesis using RevertAid Reverse Transcriptase (Thermo Fisher Scientific, Waltham, MA, USA) and specific RT primers from TaqMan ${ }^{\circledR}$ Gene Expression Assays for hsa-miR-222 (002276) and RNU44 (001094) (Thermo Fisher Scientific, Waltham, MA, USA). Real-time PCR was performed on 7900HT Fast Real-Time PCR System (Applied Biosystems, Foster City, CA, USA), using TaqMan ${ }^{\circledR}$ Gene Expression Assays for hsa-miR-222 (002276) and RNU44 (001094), as well as TaqMan ${ }^{\circledR}$ Universal Master Mix II (Applied Biosystems, Foster City, CA, USA). The level of miR-222 expression was calculated relative to RNU44 expression using the comparative ddCt method, and healthy controls as a calibrator. 


\subsection{Statistical Analysis and Definition of Clinical Endpoints}

Data is presented as medians with range or as absolute numbers with percentages. Differences in continuous variables were analyzed using the Mann-Whitney U test for distribution between two groups. Analyses of frequencies were performed using the $\chi^{2}$ test for $2 \times 2$ tables, or the Fisher exact test for larger tables. Survival analysis was performed by the Kaplan-Meier method, and differences in survival distributions were evaluated using the Log-Rank test.

Overall survival (OS) was calculated from the first day of therapy to death or last visit. Patients undergoing hematopoietic stem cell transplantation (HSCT) were censored at the time of transplantation (23 patients underwent HSCT). Disease-free survival (DFS) for patients who had achieved complete remission (CR) was measured from the date of $\mathrm{CR}$ to relapse/death/last follow-up.

The statistical analyses were performed using the $\mathrm{R}$ version 4.1.2. (R Core Team, Vienna, Austria) For all analyses, the $p$ values were 2 -tailed, and $p<0.05$ was considered statistically significant.

\section{Results}

\subsection{GAS5 Expression Level in De Novo Acute Myeloid Leukemia (AML) Patients}

The expression level of the GAS5 gene was first evaluated in a cohort of 94 AML patients: 5 patients were therapy-related AML (tAML), 14 were secondary AML (sAML), and 75 of them were de novo AML patients. Median expression level of the entire cohort of patients was $0.59(0.02-6.81)$, which was significantly lower than the level detected in healthy individuals (median 1.40, range 0.77-2.42) $(p<0.01$ ).

Moreover, in comparison to healthy controls, GAS5 expression level was lower in each of the aforementioned groups of patients individually. In tAML median expression was $0.19(0.06-1.03)(p<0.01)$, in sAML it was $0.32(0.11-3.62)(p<0.01)$ and in de novo AML it was $0.70(0.02-6.81)(p<0.01)$ (Figure 1$)$.

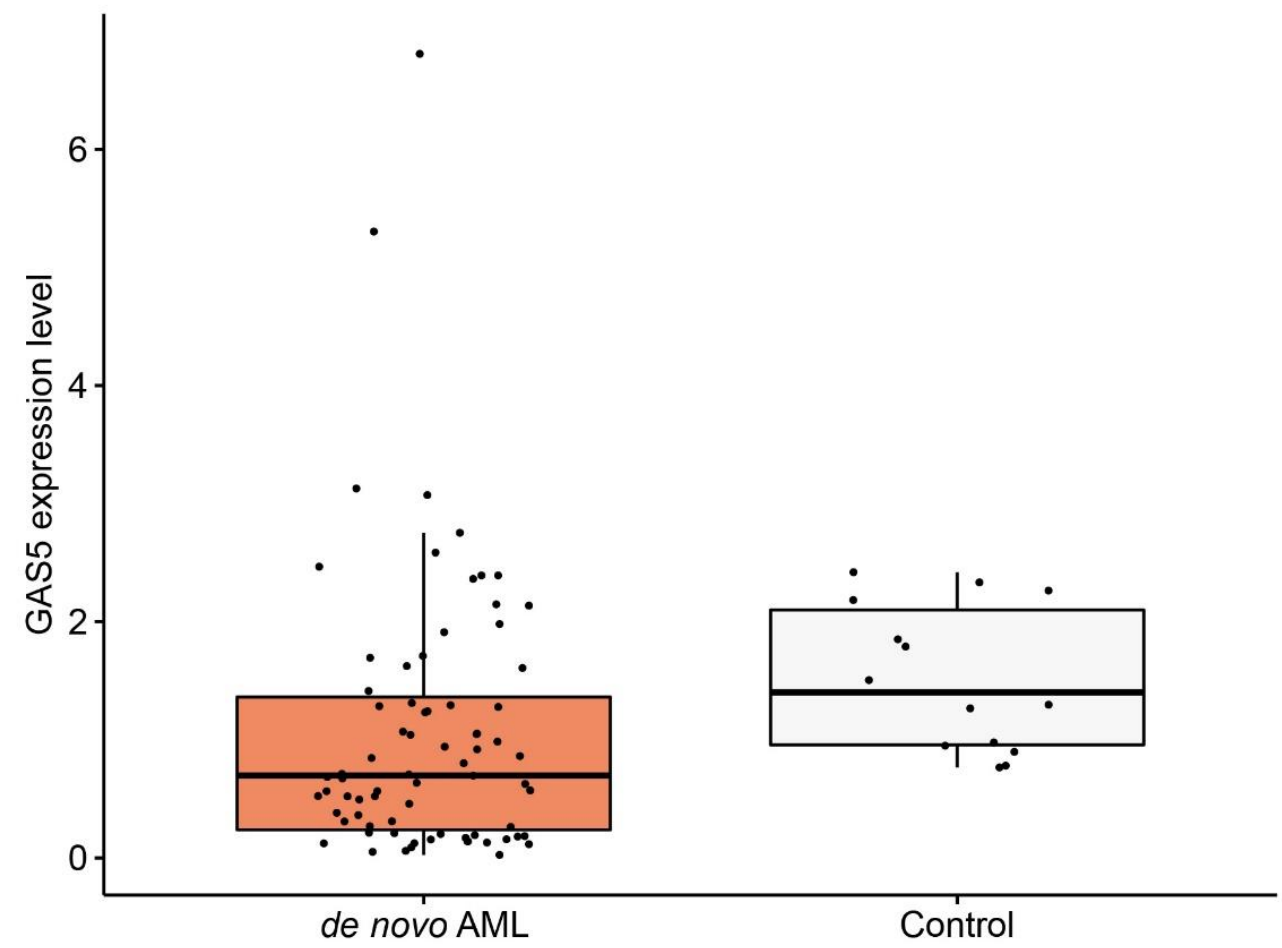

Figure 1. Box-plot representation of growth arrest-specific transcript 5 (GAS5) expression detected among de novo acute myeloid leukemia (AML) patients $(n=75)$ and healthy controls $(n=14)$. 
Due to the size of individual groups, further analysis was limited only to de novo AML patients. We used ROC curve analysis to calculate the "cut-off" value for discrimination between AML patients and healthy controls. The GAS5 expression level of 0.88 was determined as the most predictive one $(\mathrm{AUC}=0.73$, Sensitivity $=85.7 \%$, Specificity $=57.3 \%$, $p<0.01)$. Using this value, patients were divided into low GAS5 expression (GAS5 ${ }^{\text {low }}$ ) and high GAS5 expression (GAS5 $5^{\text {high }}$ ) group. At diagnosis, 58\% of patients $(43 / 75)$ had low GAS5 expression. Analyzing the association of GAS5 expression level with clinical characteristic of de novo AML patients, we found that $G A S 5^{\text {low }}$ patients had significantly higher Lactate Dehydrogenase (LDH) levels in comparison to GAS5 $5^{\text {high }}$ patients $(p=0.04)$. Also, GAS5 $5^{\text {low }}$ status was significantly associated with adverse prognosis since 15/19 (79\%) adverse risk patients had a low GAS5 expression level $(p=0.04)$ (Table 1).

Table 1. Clinical characteristics of de novo AML patients stratified by the level of GAS5 expression.

\begin{tabular}{|c|c|c|c|c|}
\hline \multirow{2}{*}{ Parameter } & \multirow{2}{*}{ Total $(n=75)$} & \multicolumn{2}{|c|}{ GAS5 Expression } & \multirow{2}{*}{$p$} \\
\hline & & $G A S 5^{\text {high }}(n=32)$ & $G A S 5^{\text {low }}(n=43)$ & \\
\hline Sex & & & & 0.78 \\
\hline Male (\%) & $42(56)$ & $19(45)$ & $23(55)$ & \\
\hline Female (\%) & $33(44)$ & $13(39)$ & $20(61)$ & \\
\hline Age, years, median (range) & $50(18-62)$ & $51(24-62)$ & $48(18-62)$ & 0.44 \\
\hline $\begin{array}{c}\text { WBC (White Blood Cells) } \\
\text { count, } \times 10^{9} / \mathrm{L} \text {, median (range) }\end{array}$ & $17.1(1-348.8)$ & $9.8(1-183.7)$ & $22.3(1.2-348.8)$ & 0.09 \\
\hline $\begin{array}{l}\text { Hemoglobin }(\mathrm{g} / \mathrm{L}) \\
\text { median (range) }\end{array}$ & $97(2-166)$ & $98.5(2-153)$ & $97(24-166)$ & 0.62 \\
\hline $\begin{array}{l}\text { Platelets }\left(\times 10^{9} / \mathrm{L}\right) \\
\text { median (range) }\end{array}$ & $54(1-422)$ & $48.5(12-216)$ & $54(1-422)$ & 0.59 \\
\hline LDH (U/L), median (range) & $287.5(153-4169)$ & $175(153-4196)$ & $462.5(2-2904)$ & 0.04 \\
\hline $\begin{array}{c}\text { Peripheral blood blast (\%) } \\
\text { median (range) }\end{array}$ & $16(0-98)$ & $13.5(0-92)$ & $21(0-98)$ & 0.43 \\
\hline $\begin{array}{l}\text { Bone marrow blasts }(\%) \\
\text { median (range) }\end{array}$ & $63(21-97)$ & $60.5(21-97)$ & $67(21-97)$ & 0.81 \\
\hline CD34 (\%) & & & & 0.31 \\
\hline present & $46(61)$ & $20(43)$ & $26(57)$ & \\
\hline absent & $22(39)$ & $6(27)$ & $16(73)$ & \\
\hline FAB (\%) & & & & 0.15 \\
\hline M0 & $6(8)$ & $3(50)$ & $3(50)$ & \\
\hline M1 & $10(13)$ & $5(50)$ & $5(50)$ & \\
\hline M2 & $17(23)$ & $10(59)$ & $7(41)$ & \\
\hline M4 & $28(37)$ & $12(43)$ & $16(57)$ & \\
\hline M5 & $14(19)$ & $2(14)$ & $12(86)$ & \\
\hline Prognostic group-ELN (\%) & & & & 0.04 \\
\hline Favorable & $12(16)$ & $4(33)$ & $8(67)$ & \\
\hline Intermediate & $44(59)$ & $24(55)$ & $20(45)$ & \\
\hline Adverse & $19(25)$ & $4(21)$ & $15(79)$ & \\
\hline Complete remission (\%) & & & & 1.0 \\
\hline success & $41(55)$ & $17(41)$ & $24(59)$ & \\
\hline failure & $34(45)$ & $15(44)$ & $19(56)$ & \\
\hline Relapse (\%) & & & & 0.38 \\
\hline yes & $22(51)$ & $11(50)$ & $11(50)$ & \\
\hline no & $19(49)$ & $6(32)$ & $13(68)$ & \\
\hline
\end{tabular}




\subsection{Prognostic Significance of GAS5 Expression Level in De Novo AML Patients}

In our cohort of de novo AML patients, the CR rate was 55\%. Among GAS5 $5^{\text {low }}$ patients induction treatment failure was more frequent compared to GAS5 ${ }^{\text {high }}$, but without significance ( $56 \%$ vs. $44 \%, p=1.0)$. Although the duration of DFS in GAS5 $5^{\text {low }}$ patients was significantly lower than in $G A S 5^{\text {high }}$ patients $(p=0.02)$, survival analysis failed to confirm this finding. Nonetheless, a trend towards lower DFS in GAS5 ${ }^{\text {low }}$ patients was evident (11 vs. 18 months median, Log-Rank $=3.2, p=0.07$ ) (Figure 2a). Similarly, the OS duration in GAS5 $^{\text {low }}$ patients was also significantly lower $(p=0.03)$, but survival analysis showed no significant difference in OS between GAS5 ${ }^{\text {low }}$ and GAS5 $5^{\text {high }}$ patients (6.3 vs. 13 months median, Log-Rank $=2.6, p=0.11$ ) (Figure 2b).

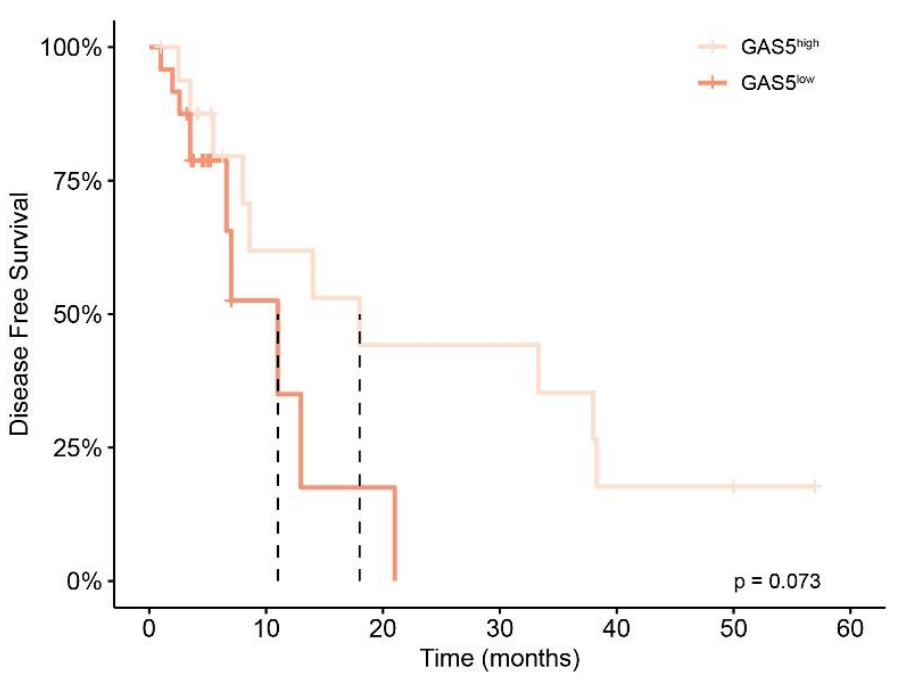

(a)

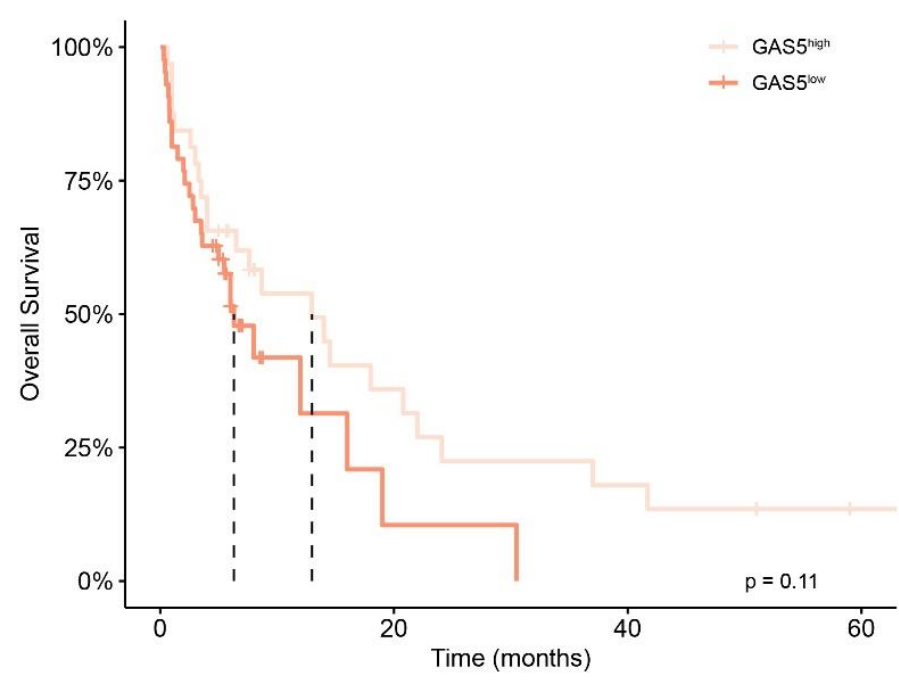

(b)

Figure 2. Kaplan-Meier analysis of disease-free survival (DFS) and overall survival (OS) in de novo AML patients according to GAS5 expression status; (a) analyses of DFS between GAS5 $5^{\text {high }}$ patients ( $n=17,6$ censored) and GAS5 $5^{\text {low }}$ patients ( $n=24,10$ censored) (11 vs. 18 months median, Log-Rank $=3.2, p=0.07)$. (b) analyses of OS between $G A S 5^{\text {high }}$ patients $(n=32,8$ censored) and GAS5 ${ }^{\text {low }}$ patients ( $n=43,13$ censored) (6.3 vs. 13 months median, Log-Rank $=2.6, p=0.11$ ).

\subsection{GAS5 Expression Level in AML-NK Patients}

Since AML-NK patients represent the largest subgroup of adult AML patients, and since it is defined as a group with intermediate risk, we wanted to examine whether the analysis of GAS5 expression can contribute to a more accurate risk stratification. Our cohort consisted of 39 AML-NK patients, and 23 of them (59\%) had GAS5 ${ }^{\text {low }}$ status (Table 2). We analyzed the association of GAS5 expression level with other molecular markers such as FLT3-ITD and NPM1 mutational status (accountable for poor and favorable prognostic influence, respectively) but we could not detect whether these mutations were significantly associated with either $G A S 5^{\text {low }}$ or GAS5 $5^{\text {high }}$ patient group ( $p=0.32$, and $p=0.21$ ) (Table 2). Survival analysis showed no statistically significant difference in DFS between GAS5 $5^{\text {low }}$ and GAS5 ${ }^{\text {high }}$ groups (11 vs. 18 months, Log-Rank $=1.6, p=0.2$ ), and similar results were obtained when we analyzed OS in these two groups ( 8 vs. 7 months, Log-Rank $=0.2$, $p=0.6)$ in AML-NK patients. 
Table 2. Mutational status of FLT3-ITD and NPM1 gene and miR-222 expression status in AML-NK patients stratified by the level of GAS5 expression.

\begin{tabular}{|c|c|c|c|c|}
\hline \multirow{2}{*}{ Parameter } & \multirow{2}{*}{ Total $(n=39)$} & \multicolumn{2}{|c|}{ GAS5 Expression } & \multirow{2}{*}{$p$} \\
\hline & & GAS5 $5^{\text {high }}(n=16)$ & $G A S 5^{\text {low }}(n=23)$ & \\
\hline FLT3-ITD mutations & & & & 0.32 \\
\hline present $(\%)$ & $12(31)$ & $3(25)$ & $9(75)$ & \\
\hline absent $(\%)$ & $27(69)$ & $13(48)$ & $14(52)$ & \\
\hline NPM1mutations & & & & 0.21 \\
\hline present $(\%)$ & $13(33)$ & $3(25)$ & $10(75)$ & \\
\hline absent $(\%)$ & $26(67)$ & $13(50)$ & $13(50)$ & \\
\hline FLT3-ITD/NPM1 status(risk group) & & & & 0.05 \\
\hline FLT3-ITD ${ }^{-}$NNPM1 $^{-}$(intermediate) & $20(51)$ & $12(60)$ & $8(40)$ & \\
\hline FLT3-ITD ${ }^{+}$(adverse) & $12(31)$ & $3(25)$ & $9(75)$ & \\
\hline $\mathrm{NPM1}^{+}$(favorable) & $7(18)$ & $1(14)$ & $6(86)$ & \\
\hline miR-222 expression status & & & & 0.40 \\
\hline high (\%) & $19(49)$ & $6(32)$ & $13(68)$ & \\
\hline low $(\%)$ & $20(51)$ & $10(50)$ & $10(50)$ & \\
\hline
\end{tabular}

\subsection{Prognostic Significance of MiR-222 Expression Level in AML-NK Patients}

To further examine the influence of GAS5 expression level on prognosis of AML-NK patients, we decided to investigate the expression level of miR-222, a micro-RNA whose oncogenic function is known to be involved in GAS5 expression and function. The median expression level of miR-222 in AML-NK patients was 5.77 (range 0.08-17.13), which was significantly higher compared to the median expression level found among healthy controls $(0.89$, range $0.76-1.47 ; p=0.01)$. Median expression level was used to divide patients into miR-222 high (miR-222 ${ }^{\text {high }}$ ) and miR-222 low (miR-222 ${ }^{\text {low }}$ ) expression groups. No significant associations were detected between miR-222 expression status and other prognostic molecular markers. Also, survival analysis showed no statistically significant difference in DFS between miR-222 ${ }^{\text {high }}$ and miR-222 ${ }^{\text {low }}$ groups (13 vs. 11 months, Log-Rank $=0.1, p=0.8$ ), nor in OS (11 vs. 14 months, Log-Rank $=0.3, p=0.6$ ) in AML-NK patients.

We detected that $68 \%$ of miR-222 $2^{\text {high }}$ patients had GAS5 $5^{\text {low }}$ status. In order to investigate the possibility of combined influence of these two ncRNAs on the prognosis and outcome of the disease, in further analysis we defined the GAS5 $5^{\text {low }} / \mathrm{miR}-222^{\text {high }}$ group and compared it with other groups that did not meet this criterion. However, survival analysis showed no statistically significant difference in DFS between the GAS5 $5^{\text {low }} / \mathrm{miR}-222^{\text {high }}$ group and other patients ( 13 vs. 11 months, Log-Rank $=0.2, p=0.7$ ). The same goes for the duration of OS within this group of patients that was not significantly different compared to others (16 vs. 6 months, Log-Rank $=0.1, p=0.8$ ).

\section{Discussion}

Although the genetic and epigenetic background and associated cytogenetic and molecular genetic markers have significantly contributed to the understanding of AML biology, as well as to improvement of diagnosis, prognostication, and monitoring of the clinical course of disease, the pathogenesis of AML still remains obscure. Consequently, the majority of AML patients cannot be cured by currently available therapy.

Therefore, many studies are conducted aiming to elucidate the relevant pathogenic mechanisms in AML. The ultimate goal is to discover novel biomarkers and therapeutic targets. In recent years non-coding RNAs, such as, microRNAs and lncRNAs have been postulated as new candidates to be considered as markers relevant for AML, since their role in gene expression regulation has been demonstrated [26-28]. Since variants in genes for non-coding RNAs are rare, alterations in their expression levels are a prominent factor governing their effects [29]. 
The role of lncRNA GAS5 and miR-222 in pathogenesis of AML has been anticipated only in a few studies [14,30]. Thus, the idea of their clinical use is even more hypothetical. Our study contributes to knowledge concerning AML in this respect. This is the first study enrolling AML patients which intends to evaluate potential clinical application of lncRNA GAS5 and miR-222 expression.

Our study included a total of 94 clearly stratified younger ( $<65$ years) AML patients, including 75 de novo AML, 14 secondary AML and 5 therapy-related AML patients. We found that, compared to healthy individuals, GAS5 expression was underexpressed in all these groups of AML patients. This is consistent with previous studies undertaken in different types of solid cancer, as well as in B lymphocytic leukemia. [17,31] This aligns with the hypothesized role of GAS5 as a tumor-suppressor lncRNA which inhibits proliferation and promotes apoptosis [8,32]. Furthermore, when we analyzed the prognostic impact of GAS5 expression among de novo AML patients we found that low GAS5 expression is predominantly detected among patients with an adverse prognosis. This relation of GAS5 $5^{\text {low }}$ status with adverse prognosis is consistent with studies investigating GAS5 in solid tumors, where decrease in GAS5 expression is associated with characteristics of advanced stage tumor, like tumor size and lymph node and distal metastases [33,34]. This adds to the idea that reduced GAS5 expression has the same effect in AML as in other types of cancer [31]. Two patients have shown higher levels of GAS5 expression compared to the other patients. While it is hard to conclude the cause of this, it should be pointed out that these patients have a complex karyotype.

In previously published studies, the prognostic impact of GAS5 expression in AML was analyzed in publicly available datasets, like the Cancer Genome Atlas, where contrary to our results, GAS5 overexpression was linked to poor cytogenetic prognosis [14]. The other study uses the GEO GSE12417 dataset and reports a link between GAS5 overexpression and shorter OS, therefore, it proposes that GAS5 may have an unexplored proto-oncogenic role. The same authors also suggest that a polymorphism in the GAS5 promoter leads to higher GAS5 expression, which in AML is associated with longer platelet recovery, a poor prognosis marker [15]. Contrary to these findings our study indicates that patients with lower GAS5 expression tend to have shorter durations of DFS, and OS, which would be more in line with the effect of low GAS5 expression in other cancers [31]. However, when we analyzed AML-NK patients separately GAS5 expression level did not significantly influence the survival duration. Also, within AML-NK group of patients GAS5 expression level was not associated with already established prognostic molecular markers, like NPM1 and FLT3-ITD mutational status. Because of the fact that our research is the first in which the impact of GAS5 expression on duration of DFS and OS in AML was directly investigated, the final conclusion about prognostic significance of GAS5 expression could be elucidated only through larger studies.

One of the better studied mechanisms of lncRNA GAS5 action is negative regulation of the oncogenic miR-222 by acting as an endogenous sponge, which was first proven in glioma [21]. The same GAS5/miR-222 axis was later shown in gastric cancer, papillary thyroid carcinoma, and most recently in B lymphocytic leukemia [19,22,23]. We analyzed the expression of miR-222 in our AML-NK group of patients and found that it was significantly overexpressed compared to healthy controls. This is in accordance with a previous study that analyzed comprehensive miRNA expression patterns in AML, as well as with studies on miR-222 in other types of cancer $[20,35]$. However, miR-222 overexpression did not show prognostic significance in our AML-NK group. This is consistent with recent investigation of miR-222 expression, based on data from the Cancer Genome Atlas that also found that high miR-222 expression did not have significant impact on prognosis, and contrary to the oncogenic role of miR-222, the authors reported that high miR-222 expression seemed to indicate a tendency towards longer survival [36]. Because of the reported negative correlation between GAS5 and miR-222 expression, we performed analysis of the combined influence of GAS5 and miR-222 expression level on prognosis in AML-NK patients. Nonetheless, survival analysis of $G A S 5^{\text {low }} / \mathrm{miR}-222^{\text {high }}$ vs. other AML-NK patients did 
not show any statistical significance. These results may be in part due to one of the major targets of miR-222 identified in other malignancies, PTEN, not being as involved in AML development as it is in other malignancies, although further studies on larger cohorts are needed for a conclusive result $[19,22,37]$.

We believe that the results obtained in our study are significant, regardless of the relatively small sample size. Our cohort of AML patients was selected to include only younger patients ( $<65$ years). In that way we tried to avoid the influence of treatment related mortality on the analysis of the prognostic significance of GAS5 and miR-222. Treatment related mortality is primarily associated with older AML patients resulting in in worse prognosis in this age group. However, overtreatment of younger AML patients is also a common problem [38]. In order to prevent treatment toxicity in younger AML patients, a more comprehensive molecular characterization of each patient individually is needed. Our cohort of patients was tested only for basic molecular markers (cytogenetics, molecular analysis for recurrent translocations and FLT3-ITD and NPM1 mutations). We suspect that our results on GAS5 and miR-222 expression pattern in younger AML patients would be more clearly defined in their effects on clinical parameters, if the analysis were to be performed in patients who were extensively characterized on a molecular level (i.e., using targeted NGS for most common leukemia-associated mutations), as well as if the impact of other significant variables was grouped in a multivariate approach.

We also believe that our study is important because of the selection of non-coding RNAs that we analyzed. Namely, there is a growing number of studies in which the importance of GAS5 and miR-222 is related to therapy response, and it has also been shown that GAS5 and miR-222 themselves can be a target for the design and application of new therapies.

Through identification of the signaling pathways related to GAS5 and miR-222 a possibility for targeted innovative therapy for AML patients has emerged. Since downregulation of GAS5 is a hallmark of different types of cancer, induction of GAS5 overexpression could aid in regression of the tumor. It was shown that overexpression of GAS5 leads to decrease of miR-544 expression by overexpression of runt-related transcription factor 3 (RUNX3), thus supporting increased activated NK cell-mediated cytotoxicity [39]. Moreover, the expression of GAS5 is related to mTOR signaling pathway, which makes it a mediator of cytotoxic and cytostatic effects of rapalogues in mantle cell lymphomas [30]. Recently, GAS5 has emerged as a potential target for cancer immunotherapy. Upregulation of its expression can increase anti-tumor response. It is indicated that GAS5 has a role in increased NK cell cytotoxicity and that it is also involved in initiation and phagophore nucleation [40]. All of this shows that GAS5 could potentially be an important therapeutic target in AML as well.

Similarly, miR-222 has become a subject of study for potential therapeutic targeting. It is well documented that miR-222-3p plays various roles in the initiation, progression, metastasis and treatment response of cancer. It is involved in the regulation of multiple tumor signaling pathways, such as PI3K/AKT, PTEN, JAK/STAT, TRPS1/ZEB1 and EMT [36]. Several studies have shown that its expression could be a target for innovative cancer therapeutic approaches [36,41-43].

In the era of next-generation medicine, treatment of hematological malignancies, including AML, will be personalized. This can be achieved by utilizing high throughput technologies to characterize patients' specific multiple "oms" landscape. Personalized treatment protocols will certainly consider non-coding RNAs as molecular markers for individualized drug selection and dosage profiling, as well as for design of novel, molecular targeted drugs. Integrated pharmacomics will open the doors to personalized oncology.

\section{Conclusions}

Recently, the role of non-coding RNAs in cancer pathogenesis has been the focus of researchers. LncRNA GAS5 and miR-222 are the most studied as potential markers clinically relevant in many malignancies. GAS5 and miR-222 can also be targets for new therapies. 
This is the first study focused on examining GAS5 and miR-222 expression pattern in AML patients. Our results showed that GAS5 expression level in younger AML patients was lower compared to healthy controls, and it was related to adverse prognosis. In the AML-NK group, patients had a higher expression of miR-222. Complete understanding of AML pathology will be achieved when the puzzle of its "oms" background is solved. There is no doubt that GAS5 and miR-222 are pieces of that puzzle. Therefore, our initial findings indicate the need for further investigation of these two non-coding RNAs, their potential roles in leukemogenesis, and the prognosis of AML patients.

Author Contributions: Conceptualization, V.G. and D.P.; Methodology, D.P. and V.G.; Formal Analysis, D.P. and V.G.; Investigation, D.P., V.G., N.T., Z.P., N.S.V.; Data Curation, D.P., V.G., N.T., B.Z., Z.P., N.S.V., S.P.; Writing—Original Draft Preparation, D.P., V.G., N.T.; Writing-Review and Editing, S.P., B.Z.; Visualization, D.P. and V.G.; Supervision, V.G. and S.P.; Project Administration, V.G. All authors have read and agreed to the published version of the manuscript.

Funding: This work has been funded by grant from the Ministry of Education, Science and Technological Development, Republic of Serbia (Grant No. 451-03-9/2021-14/ 200042).

Institutional Review Board Statement: The study was conducted according to the guidelines of the Declaration of Helsinki and approved by the Ethics Committee of the Clinical Center of Serbia, Belgrade, Serbia. (No 110/11; Date: 23 May 2019.)

Informed Consent Statement: Informed consent was obtained from all subjects involved in the study.

Data Availability Statement: The authors confirm that the data supporting the findings of this study are available within the article, further inquiries can be directed to the corresponding author.

Conflicts of Interest: The authors declare no conflict of interest. The funders had no role in the design of the study; in the collection, analyses, or interpretation of data; in the writing of the manuscript, or in the decision to publish the results.

\section{References}

1. Arber, D.A.; Orazi, A.; Hasserjian, R.; Thiele, J.; Borowitz, M.J.; Le Beau, M.M.; Bloomfield, C.D.; Cazzola, M.; Vardiman, J.W. The 2016 revision to the World Health Organization classification of myeloid neoplasms and acute leukemia. Blood 2016, 127, 2391-2405, Erratum in Blood 2016, 127, 2391-2405. [CrossRef] [PubMed]

2. Döhner, H.; Estey, E.; Grimwade, D.; Amadori, S.; Appelbaum, F.R.; Büchner, T.; Dombret, H.; Ebert, B.L.; Fenaux, P.; Larson, R.A.; et al. Diagnosis and management of AML in adults: 2017 ELN recommendations from an international expert panel. Blood 2017, 129, 424-447. [CrossRef] [PubMed]

3. Schmitz, S.U.; Grote, P.; Herrmann, B.G. Mechanisms of long noncoding RNA function in development and disease. Cell. Mol. Life Sci. 2016, 73, 2491-2509. [CrossRef] [PubMed]

4. Schmitt, A.M.; Chang, H.Y. Long Noncoding RNAs in Cancer Pathways. Cancer Cell 2016, 29, 452-463. [CrossRef] [PubMed]

5. Ma, C.; Shi, X.; Zhu, Q.; Li, Q.; Liu, Y.; Yao, Y.; Song, Y. The growth arrest-specific transcript 5 (GAS5): A pivotal tumor suppressor long noncoding RNA in human cancers. Tumor Biol. 2015, 37, 1437-1444. [CrossRef] [PubMed]

6. Williams, G.T.; Mourtada-Maarabouni, M.; Farzaneh, F. A critical role for non-coding RNA GAS5 in growth arrest and rapamycin inhibition in human T-lymphocytes. Biochem. Soc. Trans. 2011, 39, 482-486. [CrossRef]

7. Fleming, V.J.; Hay, M.S.; Harries, D.N.; Rees, D.W. Effects of nutrient deprivation and differentiation on the expression of growth-arrest genes (gas and gadd) in F9 embryonal carcinoma cells. Biochem. J. 1998, 330, 573-579. [CrossRef]

8. Pickard, M.R.; Williams, G.T. Molecular and Cellular Mechanisms of Action of Tumour Suppressor GAS5 LncRNA. Genes 2015, 6, 484-499. [CrossRef]

9. Mazar, J.; Rosado, A.; Shelley, J.; Marchica, J.; Westmoreland, T.J. The long non-coding RNA GAS5 differentially regulates cell cycle arrest and apoptosis through activation of BRCA1 and p53 in human neuroblastoma. Oncotarget 2017, 8, 6589-6607. [CrossRef]

10. Zhang, Z.; Mo, Y.-Y. Abstract 1829: Negative regulation of lncRNA GAS5 by miR-21. Mol. Cell. Biol. 2013, 20, 1558-1568. [CrossRef]

11. Vesovic, N.; Tosic, N.; Djurasevic, T.K.; Andric, Z.; Zdravkovic, D.; Pavlovic, S.; Jovanovic, D. Expression pattern of circulating long non-coding RNA GAS5 as a novel biomarker in non-small cell lung cancer patients. Arch. Med. Sci. 2020, 16, 161. [CrossRef]

12. Wang, Y.; Wu, S.; Yang, X.; Li, X.; Chen, R. Association between polymorphism in the promoter region of lncRNA GAS5 and the risk of colorectal cancer. Biosci. Rep. 2019, 39, 20190091. [CrossRef]

13. Avgeris, M.; Tsilimantou, A.; Levis, P.K.; Tokas, T.; Sideris, D.C.; Stravodimos, K.; Ardavanis, A.; Scorilas, A. Loss of GAS5 tumour suppressor lncRNA: An independent molecular cancer biomarker for short-term relapse and progression in bladder cancer patients. Br. J. Cancer 2018, 119, 1477-1486. [CrossRef] [PubMed] 
14. Zimta, A.-A.; Tomuleasa, C.; Sahnoune, I.; Calin, G.; Berindan-Neagoe, I. Long Non-coding RNAs in Myeloid Malignancies. Front. Oncol. 2019, 9, 1048. [CrossRef]

15. Yan, H.; Zhang, D.-Y.; Li, X.; Yuan, X.-Q.; Yang, Y.-L.; Zhu, K.-W.; Zeng, H.; Li, X.-L.; Cao, S.; Zhou, H.-H.; et al. Long non-coding RNA GAS5 polymorphism predicts a poor prognosis of acute myeloid leukemia in Chinese patients via affecting hematopoietic reconstitution. Leuk. Lymphoma 2017, 58, 1948-1957. [CrossRef] [PubMed]

16. Ketab, F.N.G.; Gharesouran, J.; Ghafouri-Fard, S.; Dastar, S.; Mazraeh, S.A.; Hosseinzadeh, H.; Moradi, M.; Javadlar, M.; Hiradfar, A.; Rezamand, A.; et al. Dual biomarkers long non-coding RNA GAS5 and its target, NR3C1, contribute to acute myeloid leukemia. Exp. Mol. Pathol. 2020, 114, 104399. [CrossRef]

17. Rodriguez, P.; Paculova, H.; Kogut, S.; Heath, J.; Schjerven, H.; Frietze, S. Non-Coding RNA Signatures of B-Cell Acute Lymphoblastic Leukemia. Int. J. Mol. Sci. 2021, 22, 2683. [CrossRef]

18. Gasic, V.; Stankovic, B.; Zukic, B.; Janic, D.; Dokmanovic, L.; Krstovski, N.; Lazic, J.; Milosevic, G.; Lucafò, M.; Stocco, G.; et al Expression pattern of long non-coding RNA growth arrest-specific 5 in the remission induction therapy in childhood acute lymphoblastic leukemia. J. Med. Biochem. 2019, 38, 292-298. [CrossRef] [PubMed]

19. Zhang, X.-F.; Ye, Y.; Zhao, S.-J. LncRNA Gas5 acts as a ceRNA to regulate PTEN expression by sponging miR-222-3p in papillary thyroid carcinoma. Oncotarget 2017, 9, 3519-3530. [CrossRef] [PubMed]

20. Isken, F.; Steffen, B.; Merk, S.; Dugas, M.; Markus, B.; Tidow, N.; Zühlsdorf, M.; Illmer, T.; Thiede, C.; Berdel, W.E.; et al. Identification of acute myeloid leukaemia associated microRNA expression patterns. Br. J. Haematol. 2007, 140, 153-161. [CrossRef]

21. Zhao, X.; Wang, P.; Liu, J.; Zheng, J.; Liu, Y.; Chen, J.; Xue, Y. Gas5 Exerts Tumor-suppressive Functions in Human Glioma Cells by Targeting miR-222. Mol. Ther. 2015, 23, 1899-1911. [CrossRef] [PubMed]

22. Li, Y.; Gu, J.; Lu, H. The GAS5/miR-222 Axis Regulates Proliferation of Gastric Cancer Cells Through the PTEN/Akt/mTOR Pathway. Dig. Dis. Sci. 2017, 62, 3426-3437. [CrossRef]

23. Jing, Z.; Gao, L.; Wang, H.; Chen, J.; Ben Nie, B.; Hong, Q. Long non-coding RNA GAS5 regulates human B lymphocytic leukaemia tumourigenesis and metastasis by sponging miR-222. Cancer Biomark. 2019, 26, 385-392. [CrossRef] [PubMed]

24. Kuzmanovic, M.; Tosic, N.; Colovic, N.; Karan-Djurasevic, T.; Spasovski, V.; Radmilovic, M.; Nikcevic, G.; Suvajdzic-Vukovic, N.; Tomin, D.; Vidovic, A.; et al. Prognostic Impact ofNPM1Mutations in Serbian Adult Patients with Acute Myeloid Leukemia. Acta Haematol. 2012, 128, 203-212. [CrossRef] [PubMed]

25. Budczies, J.; Klauschen, F.; Sinn, B.V.; Gyorffy, B.; Schmitt, W.D.; Darb-Esfahani, S.; Denkert, C. Cutoff Finder: A Comprehensive and Straightforward Web Application Enabling Rapid Biomarker Cutoff Optimization. PLoS ONE 2012, 7, e51862. [CrossRef]

26. Liu, Y.; Gao, X.; Tian, X. High expression of long intergenic non-coding RNA LINC00662 contributes to malignant growth of acute myeloid leukemia cells by upregulating ROCK1 via sponging microRNA-340-5p. Eur. J. Pharmacol. 2019, 859, 172535. [CrossRef] [PubMed]

27. Liu, Y.; Sun, P.; Zhao, Y.; Liu, B. The role of long non-coding RNAs and downstream signaling pathways in leukemia progression. Hematol. Oncol. 2021, 39, 27-40. [CrossRef]

28. Wang, X.; Chen, H.; Bai, J.; He, A. MicroRNA: An important regulator in acute myeloid leukemia. Cell Biol. Int. 2017, 41, 936-945. [CrossRef]

29. Diederichs, S.; Haber, D.A. Sequence Variations of MicroRNAs in Human Cancer: Alterations in Predicted Secondary Structure Do Not Affect Processing. Cancer Res. 2006, 66, 6097-6104. [CrossRef]

30. Rommer, A.; Steinleitner, K.; Hackl, H.; Schneckenleithner, C.; Engelmann, M.; Scheideler, M.; Vlatkovic, I.; Kralovics, R.; Cerny-Reiterer, S.; Valent, P.; et al. Overexpression of primary microRNA 221/222 in acute myeloid leukemia. BMC Cancer 2013, 13, 364. [CrossRef]

31. Goustin, A.S.; Thepsuwan, P.; Kosir, M.A.; Lipovich, L. The Growth-Arrest-Specific (GAS)-5 Long Non-Coding RNA: A Fascinating lncRNA Widely Expressed in Cancers. Non-Coding RNA 2019, 5, 46. [CrossRef]

32. Mourtada-Maarabouni, M.; Hasan, A.M.; Farzaneh, F.; Williams, G.T. Inhibition of Human T-Cell Proliferation by Mammalian Target of Rapamycin (mTOR) Antagonists Requires Noncoding RNA Growth-Arrest-Specific Transcript 5 (GAS5). Mol. Pharmacol. 2010, 78, 19-28. [CrossRef] [PubMed]

33. Li, J.; Huang, H.; Li, Y.; Li, L.; Hou, W.; You, Z. Decreased expression of long non-coding RNA GAS5 promotes cell proliferation, migration and invasion, and indicates a poor prognosis in ovarian cancer. Oncol. Rep. 2016, 36, 3241-3250. [CrossRef]

34. Bian, D.; Shi, W.; Shao, Y.; Li, P.; Song, G. Long non-coding RNA GAS5 inhibits tumorigenesis via miR-137 in melanoma. Am. J. Transl. Res. 2017, 9, 1509-1520. [PubMed]

35. Ravegnini, G.; Cargnin, S.; Sammarini, G.; Zanotti, F.; Bermejo, J.L.; Hrelia, P.; Terrazzino, S.; Angelini, S. Prognostic Role of miR-221 and miR-222 Expression in Cancer Patients: A Systematic Review and Meta-Analysis. Cancers 2019, 11, 970. [CrossRef]

36. Wang, D.; Sang, Y.; Sun, T.; Kong, P.; Zhang, L.; Dai, Y.; Cao, Y.; Tao, Z.; Liu, W. Emerging roles and mechanisms of microRNA222-3p in human cancer (Review). Int. J. Oncol. 2021, 58, 20. [CrossRef]

37. Morotti, A.; Panuzzo, C.; Crivellaro, S.; Carrá, G.; Torti, D.; Guerrasio, A.; Saglio, G. The role of PTEN in myeloid malignancies. Hematol. Rep. 2015, 7, 5844. [CrossRef] [PubMed]

38. Seval, G.C.; Ozcan, M. Treatment of Acute Myeloid Leukemia in Adolescent and Young Adult Patients. J. Clin. Med. 2015, 4, 441-459. [CrossRef] 
39. Fang, P.; Xiang, L.; Chen, W.; Li, S.; Huang, S.; Li, J.; Zhuge, L.; Jin, L.; Feng, W.; Chen, Y.; et al. LncRNA GAS5 enhanced the killing effect of NK cell on liver cancer through regulating miR-544/RUNX3. Innate Immun. 2019, 25, 99-109. [CrossRef]

40. Varier, K.M.; Dhandapani, H.; Liu, W.; Song, J.; Wang, C.; Hu, A.; Ben-David, Y.; Shen, X.; Li, Y.; Gajendran, B. An immunotherapeutic approach to decipher the role of long non-coding RNAs in cancer progression, resistance and epigenetic regulation of immune cells. J. Exp. Clin. Cancer Res. 2021, 40, 242. [CrossRef]

41. Zhai, S.; Xu, Z.; Xie, J.; Zhang, J.; Wang, X.; Peng, C.; Li, H.; Chen, H.; Shen, B.; Deng, X. Epigenetic silencing of LncRNA LINC00261 promotes c-myc-mediated aerobic glycolysis by regulating miR-222-3p/HIPK2/ERK axis and sequestering IGF2BP1. Oncogene 2021, 40, 277-291. [CrossRef] [PubMed]

42. Li, B.; Lu, Y.; Wang, H.; Han, X.; Mao, J.; Li, J.; Yu, L.; Wang, B.; Fan, S.; Yu, X.; et al. miR-221/222 enhance the tumorigenicity of human breast cancer stem cells via modulation of PTEN/Akt pathway. Biomed. Pharmacother. 2016, 79, 93-101. [CrossRef] [PubMed]

43. Panneerselvam, J.; Srivastava, A.; Muralidharan, R.; Wang, Q.; Zheng, W.; Zhao, L.; Chen, A.; Zhao, Y.D.; Munshi, A.; Ramesh, R. IL-24 modulates the high mobility group (HMG) A1/miR222 /AKT signaling in lung cancer cells. Oncotarget 2016, 7, 70247-70263. [CrossRef] [PubMed] 